\title{
Precision medicine technology hype or reality? The example
}

\section{of computer-guided dosing [version 1; peer review: 2}

\section{approved]}

\author{
Thomas M. Polasek (Di)1-3, Sepehr Shakib³,4, Amin Rostami-Hodjegan 1,5 \\ ${ }^{1}$ Certara, 100 Overlook Center, Suite 101, Princeton, NJ, 08540, USA \\ ${ }^{2}$ Centre for Medicines Use and Safety, Monash University, Melbourne, Victoria, Australia \\ ${ }^{3}$ Department of Clinical Pharmacology, Royal Adelaide Hospital, Adelaide, South Australia, Australia \\ ${ }^{4}$ Discipline of Pharmacology, Adelaide Medical School, University of Adelaide, Adelaide, Australia \\ ${ }^{5}$ Centre for Applied Pharmacokinetic Research, University of Manchester, Oxford Road, Manchester, M13 9PL, UK
}

V1 First published: 01 Oct 2019, 8:1709

https://doi.org/10.12688/f1000research.20489.1

Latest published: 17 Dec 2019, 8:1709

https://doi.org/10.12688/f1000research.20489.2

\section{Abstract}

Novel technologies labelled as 'precision medicine' are targeting all aspects of clinical care. Whilst some technological advances are undeniably exciting, many doctors at the frontline of healthcare view precision medicine as being out of reach for their patients. Computerguided dosing is a precision medicine technology that predicts drug concentrations and drug responses based on individual patient characteristics. In this opinion piece, the example of computer-guided dosing is used to illustrate eight features of a precision medicine technology less likely to be hyperbole and more likely to improve patient care. Positive features in this regard include: (1) fitting the definition of 'precision medicine'; (2) addressing a major clinical problem that negatively impacts patient care; (3) a track record of high-quality medical science published via peer-reviewed literature; (4) well-defined clinical cases for application; (5) quality evidence of benefits measured by various clinical, patient and health economic endpoints; (6) strong economic drivers; (7) user friendliness, including easy integration into clinical workflow, and (8) recognition of importance by patients and their endorsement for broader clinical use. Barriers raised by critics of the approach are given to balance the view. The value of computer-guided dosing will be decided ultimately by the extent to which it can improve cost-effective patient care.

\section{Keywords}

Precision medicine, precision dosing, computer-guided dosing, modelinformed precision dosing, personalized medicine, individualized drug therapy

\section{Open Peer Review}

Approval Status

1

2

version 2

(revision)

17 Dec 2019

version 1

01 Oct 2019

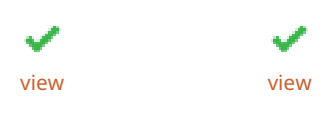

1. Daniel F. B. Wright ID, University of Otago,

Dunedin, New Zealand

2. Richard N. Upton ID, University of South

Australia, Adelaide, Australia

Any reports and responses or comments on the article can be found at the end of the article. 
Corresponding author: Thomas M. Polasek (tom.polasek@certara.com)

Author roles: Polasek TM: Conceptualization, Writing - Original Draft Preparation, Writing - Review \& Editing; Shakib S: Writing Review \& Editing; Rostami-Hodjegan A: Writing - Review \& Editing

Competing interests: TMP and ARH are employed by Certara, a company that develops and supplies modelling and simulation software and services to the pharmaceutical industry. SS has no competing interests to declare.

Grant information: The author(s) declared that no grants were involved in supporting this work.

Copyright: @ 2019 Polasek TM et al. This is an open access article distributed under the terms of the Creative Commons Attribution License, which permits unrestricted use, distribution, and reproduction in any medium, provided the original work is properly cited.

How to cite this article: Polasek TM, Shakib S and Rostami-Hodjegan A. Precision medicine technology hype or reality? The example of computer-guided dosing [version 1; peer review: 2 approved] F1000Research 2019, 8:1709

https://doi.org/10.12688/f1000research.20489.1

First published: 01 Oct 2019, 8:1709 https://doi.org/10.12688/f1000research.20489.1 


\section{Introduction}

Precision medicine is defined as 'treatments targeted to the needs of individual patients on the basis of genetic, biomarker, phenotypic, or psychosocial characteristics that distinguish a given patient from other patients with similar clinical presentations" ${ }^{1}$. Novel technologies labelled as precision medicine are targeting all aspects of clinical care with the promise of better healthcare for all via better treatment of the individual. Examples are diverse, but include companion molecular diagnostics for pharmaco- and immuno-therapy in oncology and hematology ${ }^{2}$, pharmacogenomic-guided drug and dose selection $^{3}$, and artificial intelligence to stratify clinical risk and treatment options (IBM Watson Health). Whilst some technological advances are undeniably exciting, widespread clinical application beyond specialized centers is limited, particularly outside the United States. No doubt, the words 'precision medicine' create business opportunities, advance academic careers, have political appeal, and resonate with the media and public. However, many doctors at the frontline of healthcare view precision medicine as merely indulgent fine-tuning for a privileged few rather than a 'game-changer' for all. In this opinion piece, the example of computer-guided dosing, sometimes called clinical pharmacometrics and/or model-informed precision dosing (MIPD) ${ }^{4,5}$, is used to illustrate eight features of a precision medicine technology less likely to be hyperbole and more likely to improve patient care. Barriers raised by critics of the approach are also given to balance the view.

\section{(1) Defined as precision medicine}

First, the novel technology should fit the definition of precision medicine quoted above $^{1}$, rather than just applying common sense more specifically to an individual patient e.g., fitness devices linked to applications that track and encourage exercise. Computer-guided dosing predicts drug concentrations ('exposure') in the body based on individual patient characteristics such as age, weight and gender. Some models also incorporate physiological and molecular characteristics, including drug metabolizing enzyme and transporter activities and how these change in disease states or in the presence of interacting drugs. More sophisticated models predict drug responses based on dose-exposure-response relationships, although such modelling is relatively less advanced compared with exposure and requires further understanding of many pathophysiological states e.g., disease progression models etc. The dose required for each patient to achieve the target exposure is then relatively straightforward to determine, with the ultimate goal of accurately predicting drug responses. In cases where the pathophysiology is relatively simple or well understood (e.g., bacterial cell killing and antiviral effects), prediction of drug responses is relatively advanced ${ }^{6}$. Critics of the approach use examples where the pathophysiology is complex or poorly understood (e.g., neurology and psychiatry), which makes accurate modelling of drug responses difficult. Models can give dose predictions prior to starting drug treatment, but they are particularly powerful for dose adjustment via Bayesian feedback after initial drug exposure and/or a biomarker of response is known in a particular patient ${ }^{7}$.

\section{(2) Addresses a clinical problem}

The novel technology should target a well-defined clinical problem that negatively impacts patient care. Many patients receive no benefits from drug treatment. Worse still, drugs cause patient harm, costing about US $\$ 42$ billion per year globally ${ }^{8}$. One of the key reasons for these problems is that drug exposure may vary more than 10-fold for the same drug at the same dose in different patients. Computer-guided dosing adjusts for between-patient variability in drug exposure. The prescribing focus changes from selecting a dose, to selecting a dose needed to achieve a target exposure, which is one-step closer to response ${ }^{9}$. This goes against the industry culture of 'one-dose-fits-all', which is adopted for commercial reasons. Some prescribers may also underplay the role of dose as a cause of patient harm. It is accepted that dose is just one of many factors that contribute to adverse drug effect susceptibility, including Immunological, Genetic, demographic (Age and Sex), Physiological, Exogenous factors (e.g., drugdrug interactions) and Disease and disorders (e.g., renal failure), giving the mnemonic I GASPED ${ }^{10}$. But amongst these factors, dose is the major one that can, and therefore should, be modified. Thus, the clinical problem of patient harm from drugs is more likely to be reduced, rather than 'solved' by computer-guided dosing.

\section{(3) Track record of high-quality medical science}

The novel technology should not, on closer review, be so novel. Computer-guided dosing was first proposed in 1969 for anticoagulation $^{11}$, with seminal publications demonstrating clinical utility in the 1970 s for digoxin ${ }^{12}$. Since the millennium, affordable '-omics' technologies (genomics, proteomic and metabolomics), superior analysis of biological samples, improved medical imaging, and powerful computers to analyze data, have enabled many sources of between patient variability in drug exposure and/or response to be identified, understood and then modelled e.g., warfarin. A clear narrative in the peer-reviewed literature adds confidence that a precision medicine technology is not just a 'flash-in-the-pan'. However, a down-side of peer-reviewed literature is an over-emphasis of success stories, and the field of computer-guided dosing is probably also influenced by this publication bias.

\section{(4) Clinical use is well-defined}

The novel technology should have a well-defined clinical application. A classic sign of precision medicine hype is the 'oversell', usually from a commercial provider, which occurs when potential clinical utilities are promoted beyond the scope of actual clinical utilities. Computer-guided dosing is yet to be subject to such promotion, although several private companies have entered the market in recent years. Indeed, many doctors are unaware of computer-guided dosing or the clinical cases for which the approach is helpful. It is important 
to note that the patient, disease and drug characteristics that combine for high impact computer-guided dosing are well-defined ${ }^{4}$. The approach is best for difficult to dose drugs in difficult to dose patients when the clinical stakes are high. In other words, the use of narrow therapeutic index drugs when safer options are unavailable/unacceptable in pregnant women, neonates, children, those with severe organ dysfunction, the hemodynamically unstable, the frail elderly, and patients with multiple co-morbidities on polypharmacy. These patient groups are typically excluded from clinical trials that establish the recommended dose(s), so doctors are 'flying blind' with dosing when they must use drug treatment. For precision medicine technologies, knowing which patients, which diseases, and which drugs not to study is equally as important as knowing which clinical cases to study. This allows efficient resource allocation and the evidence of clinical utility to grow more rapidly. An important criticism of precision medicine is that it is not a 'game-changer' for all, however, all significant changes in clinical practice have begun in a narrow and clearly defined group of patients.

\section{(5) Evidence of benefits}

The novel technology should be supported by several independent studies that include a range of clinical, patient-reported and health economic endpoints. For computer-guided dosing, there is work on clinical outcomes with antibiotics in the critically ill, with immunosuppressants and chemotherapy in serious pediatric illnesses, and with chemotherapy in adult oncology. For example, recent randomized controlled studies comparing computer-guided dosing of paclitaxel versus body surface area-based dosing in patients with non-small cell lung cancer show trends for decreased toxicities (e.g., grade 4 hematological toxicities and neutropenia and $>$ grade 2 neuropathy) without compromising efficacy ${ }^{13,14}$. More high quality studies of this nature are required to generate clinical evidence supportive of computer-guided dosing more broadly ${ }^{15}$. Data are especially needed for commonly used narrow therapeutic index drugs, including those started by medical specialists and continued by general practitioners, such as the direct oral anticoagulants and psychotropics (clozapine, lithium).

\section{(6) Strong economic drivers}

The novel technology should catch the eye of business developers and healthcare administrators. In the last two decades, computer-guided dosing has revolutionized drug development because it saves the pharmaceutical industry time and money ${ }^{7,16}$. The appeal of translating this success to healthcare is the economic driver for the private sector. Unsustainable public spending on healthcare is shifting the re-imbursement of drugs from a supply-based model to one that rewards positive clinical outcomes. This is forcing a re-think on the 'one-dosefits-all' strategy that has previously served drug developers well. Publicly funded incentives would then be in place to find the best drug at the best dose for a particular patient. Unfortunately, strong economic drivers also create an environment for 'sharks in the water', further emphasizing the need for high quality evidence of benefits (section 5).

\section{(7) User friendliness}

The novel technology should be embraced by doctors. Disruption to clinical workflow should be minimal and considered 'worth the effort' i.e., the sweet spot between clinical speed and clinical accuracy is retained. Decision support tools (DSTs) for computer-guided dosing have been integrated successfully into various in-house and commercially available electronic health records. An example with high doctor satisfaction is a DST built for busulfan, a narrow therapeutic antineoplastic drug used to prepare pediatric patients for bone marrow transplantation ${ }^{17}$. However, such examples are rare, and a major barrier to computer-guided dosing will be familiarity and acceptance of DSTs by everyday prescribers who remain unconvinced of the clinical need. A 'forcing function' for adoption may be the automatic inclusion of such tools within e-prescribing modules of commercially available electronic health records.

\section{(8) Patients drive broader clinical uptake}

Finally, the novel technology should be embraced by patients and their advocates. Broader and faster uptake of technology occurs when 'consumers' experience benefits and spread the word. Indeed, some precision medicine developers are now by-passing the medical profession entirely with direct-toconsumer strategies e.g., pharmacogenomic testing. There are no published data thus far on the appeal of computer-guided dosing to patients, so future studies should include endpoints to capture their perspectives ${ }^{5}$.

\section{Conclusion}

Many doctors resent the insinuation of precision medicine that their clinical practice is not individualized enough and therefore not good enough. The disparity between innovative clinical applications and the reality of resource-constrained clinical practice is considered too wide. Features of precision medicine technologies more likely to bridge this disparity are illustrated here using the example of computer-guided dosing. This approach is now 50 years old and has matured to the point where clinical implementation is expected beyond specialist units in academic medical centers. The value of disruptive technologies in clinical medicine, including computer-guided dosing, will be decided ultimately by the extent to which they can improve cost-effective patient care.

\section{Acknowledgements}

We thank Gillian E. Caughey, BSc (Hons), PhD and Nicholas Farinola, BMedSci, BMBS, FRACP from the Department of Clinical Pharmacology, Royal Adelaide Hospital, and the University of Adelaide, for their valuable insights and comments, for which they received no compensation. 
1. Jameson JL, Longo DL: Precision medicine--personalized, problematic, and promising. N Engl J Med. 2015; 372(23): 2229-2234.

PubMed Abstract | Publisher Full Text

2. Polasek TM, Ambler K, Scott HS, et al.: Targeted pharmacotherapy after somatic cancer mutation screening [version 2; peer review: 2 approved]. F1000Res. 2016; 5: 1551.

PubMed Abstract | Publisher Full Text | Free Full Text

3. Polasek TM, Mina K, Suthers G: Pharmacogenomics in general practice: The time has come. Aust J Gen Pract. 2019; 48(3): 100-105. PubMed Abstract

4. Darwich AS, Ogungbenro K, Vinks AA, et al:: Why has model-informed precision dosing not yet become common clinical reality? lessons from the past and a roadmap for the future. Clin Pharmacol Ther. 2017; 101(5): 646-656. PubMed Abstract | Publisher Full Text

5. Polasek TM, Shakib S, Rostami-Hodjegan A: Precision dosing in clinical medicine: present and future. Expert Rev Clin Pharmacol. 2018; 11(8): 743-746. PubMed Abstract | Publisher Full Text

6. Kamal MA, Smith PF, Chaiyakunapruk N, et al:: Interdisciplinary pharmacometrics linking oseltamivir pharmacology, influenza epidemiology and health economics to inform antiviral use in pandemics. $\mathrm{Br} \mathrm{J} \mathrm{Clin}$ Pharmacol. 2017; 83(7): 1580-1594.

PubMed Abstract | Publisher Full Text | Free Full Text

7. Polasek TM, Rayner CR, Peck RW, et al:: Toward Dynamic Prescribing Information: Codevelopment of Companion Model-Informed Precision Dosing Tools in Drug Development. Clin Pharmacol Drug Dev. 2019; 8(4): 418-425. PubMed Abstract | Publisher Full Text

8. World Health Organization: Medication Without Harm - Global Patient Safety Challenge on Medication Safety. Geneva, 2017. Reference Source

9. Peck RW: Precision medicine is not just genomics: the right dose for every patient. Annu Rev Pharmacol Toxicol. 2018; 58: 105-122. PubMed Abstract | Publisher Full Text
10. Ferner R, Aronson J: Susceptibility to adverse drug reactions. Br J Clin Pharmacol. 2019.

PubMed Abstract | Publisher Full Text

11. Sheiner LB: Computer-aided long-term anticoagulation therapy. Comput Biomed Res. 1969; 2(6): 507-518.

PubMed Abstract | Publisher Full Text

12. Peck CC, Sheiner LB, Martin CM, et al:: Computer-assisted digoxin therapy. N Engl J Med. 1973; 289(9): 441-446.

PubMed Abstract | Publisher Full Text

13. Joerger M, von Pawel J, Kraff S, et al:: Open-label, randomized study of individualized, pharmacokinetically (PK)-guided dosing of paclitaxel combined with carboplatin or cisplatin in patients with advanced non-small-cell lung cancer (NSCLC). Ann Oncol. 2016; 27(10): 1895-1902. PubMed Abstract | Publisher Full Text

14. Zhang J, Zhou F, Qi H, et al:: Randomized study of individualized pharmacokinetically-guided dosing of paclitaxel compared with body-surface area dosing in Chinese patients with advanced non-small cell lung cancer. $\mathrm{Br}$ Clin Pharmacol. 2019. Clin Pharmacol. 2019.
PubMed Abstract | Publisher Full Text

15. Wright DFB, Martin JH, Cremers S: Spotlight Commentary: Model-informed precision dosing must demonstrate improved patient outcomes. $\mathrm{Br} \mathrm{J}$ Clin Pharmacol. 2019.

PubMed Abstract | Publisher Full Text

16. Polasek TM, Rostami-Hodjegan A, Yim DS, et al.: What Does it Take to Make Model-Informed Precision Dosing Common Practice? Report from the 1st Asian Symposium on Precision Dosing. AAPS J. 2019; 21(2): 17. PubMed Abstract | Publisher Full Text

17. Abdel-Rahman SM, Breitkreutz ML, Bi C, et al:: Design and testing of an EHRintegrated, busulfan pharmacokinetic decision support tool for the point-ofcare clinician. Front Pharmacol. 2016; 7: 65.

PubMed Abstract | Publisher Full Text | Free Full Text 


\section{Open Peer Review}

\section{Current Peer Review Status:}

\section{Version 1}

Reviewer Report 11 November 2019

https://doi.org/10.5256/f1000research.22521.r55228

(C) 2019 Upton R. This is an open access peer review report distributed under the terms of the Creative Commons Attribution License, which permits unrestricted use, distribution, and reproduction in any medium, provided the original work is properly cited.

\section{Richard N. Upton}

School of Pharmacy and Medical Sciences, University of South Australia, Adelaide, SA, Australia

This seems a sensible opinion article discussing the requirements for the successful implementation of precision medicine using drug dosing as an example.

Two minor comments.

1. Under Introduction heading (1), bacterial cell killing and antiviral effects are not examples of pathophysiologies. These are examples of mechanisms of action

2. Under Introduction heading (6), "Unsustainable public spending on healthcare ": Why public here? Private spending via insurance can also be unsustainable.

Is the topic of the opinion article discussed accurately in the context of the current literature?

Yes

Are all factual statements correct and adequately supported by citations?

Yes

Are arguments sufficiently supported by evidence from the published literature? Yes

Are the conclusions drawn balanced and justified on the basis of the presented arguments? Yes

Competing Interests: I have been involved in the development of precision dosing software and have been paid for this work.

Reviewer Expertise: Pharmacometrics, Precision Medicine 


\section{I confirm that I have read this submission and believe that I have an appropriate level of expertise to confirm that it is of an acceptable scientific standard.}

\section{Author Response 17 Nov 2019}

Thomas Polasek, Certara, 100 Overlook Center, Suite 101, Princeton, USA

We thank Professor Upton for reviewing our manuscript. We have now uploaded a revised copy of the manuscript with tracked changes based on the comments.

\section{Comment 1}

Under Introduction heading (1), bacterial cell killing and antiviral effects are not examples of pathophysiologies. These are examples of mechanisms of action.

\section{Response}

We thank Professor Upton for picking up this mistake. The word 'pathophysiology' has been replaced with 'mechanisms of drug action' as suggested.

\section{Comment 2}

Under Introduction heading (6), "Unsustainable public spending on healthcare ": Why public here? Private spending via insurance can also be unsustainable.

\section{Response}

We thank Professor Upton for this comment. We have now added private spending to this paragraph.

Competing Interests: TMP and ARH are both employees of Certara, a global company that provides pharmacometric software and services to the pharmaceutical industry.

Reviewer Report 30 October 2019

https://doi.org/10.5256/f1000research.22521.r54521

(C) 2019 Wright D. This is an open access peer review report distributed under the terms of the Creative Commons Attribution License, which permits unrestricted use, distribution, and reproduction in any medium, provided the original work is properly cited.

\section{Daniel F. B. Wright}

School of Pharmacy, University of Otago, Dunedin, New Zealand

This commentary provides a well-argued and timely overview of important features of precision medicine, specifically focused on computer guided dosing. This is an important contribution at a time when model-informed precision-dosing is being touted (once again, or 'finally' depending on your perspective) as a means of improving patient outcomes and mitigating drug related harm. This argument is not new, but the well-considered points raised by the authors suggest that the conversation around this topic is ready to be escalated. Indeed, there is a general sense in the 
literature - whether real or not - that proponents of this approach are making headway with broad clinical implementation in some settings.

It is important to emphasize that computer-guided dosing - as used throughout the paper - is a very broad term. Outputs of clinical pharmacometric analyses or model-informed precision dosing are not synonymous with computer based dosing, as implied by the authors in the introduction, but are simply examples. Almost anything can be put on a computer as part of decision-support system, including simple empirical dosing guidelines that may have been developed historically from less than robust analyses. The authors address this point somewhat under "(1) Defined as precision medicine" but I wonder if a clear statement indicating that the paper is referring to dosing guidance that has been derived from (hopefully) robust modelling analysis is warranted. These could be simple model-based dosing tables implemented in e-prescribing systems or purpose-built Bayesian feedback programmes. As the authors note, the models underpinning the outputs might be population pharmacokinetic models or mechanistic PBPK or systems pharmacology models (there are currently few examples of the latter).

On a related note, the statement under "(2) addressed a clinical problem" that computer-guided dosing will adjust for between patient variability in drug exposure is not necessarily true. This is only the case of the dosing tool has been derived from a modelling analysis and/or is implemented in a Bayesian feedback system.

The title seems to promise some debate about whether precision medicine is hype or reality. While the paper presents eight characteristics for precision medicine that are required for "reality", i.e. to inform clinical care, there is only a passing reference to the hype. The title might need some consideration to ensure that it aligns with the content of the paper.

A minor point. The eight features are nicely presented but (1) and (3) could be relabelled for clarity. "Defined as precision medicine" is a bit hard to understand on first read. Something like, for example, (1) "aligns to an accepted definition of precision medicine", (3) "Derived from highquality medicine science" are suggestions.

Is the topic of the opinion article discussed accurately in the context of the current literature?

Yes

Are all factual statements correct and adequately supported by citations? Yes

Are arguments sufficiently supported by evidence from the published literature? Yes

Are the conclusions drawn balanced and justified on the basis of the presented arguments? Yes

Competing Interests: I am planning to co-author a paper with one of the authors in the future.

Reviewer Expertise: Model-informed precision dosing, pharmacometrics, clinical pharmacology 


\section{I confirm that I have read this submission and believe that I have an appropriate level of expertise to confirm that it is of an acceptable scientific standard.}

\section{Author Response 17 Nov 2019}

Thomas Polasek, Certara, 100 Overlook Center, Suite 101, Princeton, USA

We thank Dr Wright for reviewing our manuscript and commenting on our work. We have now uploaded a revised copy of the manuscript with tracked changes based on the suggestions. Indeed, the revised version has benefited greatly from this input. Individual responses to each of the major comments are provided below.

\section{Comment 1}

It is important to emphasize that computer-guided dosing - as used throughout the paper is a very broad term. Outputs of clinical pharmacometric analyses or model-informed precision dosing are not synonymous with computer based dosing, as implied by the authors in the introduction, but are simply examples.

\section{Response}

We thank Dr Wright for this suggestion and we agree. The description 'computer-guided dosing' was originally used instead of 'model-informed precision dosing (MIPD)' to appeal to a general audience less familiar with modelling and simulation in clinical pharmacology. However, after reading the review we concur that the more accurate description is superior, more focused, and less misleading. Thus, we have changed 'computer-guided dosing' throughout the paper to 'model-informed precision dosing', including in the title.

\section{Comment 2}

the statement under "(2) addressed a clinical problem" that computer-guided dosing will adjust for between patient variability in drug exposure is not necessarily true. This is only the case of the dosing tool has been derived from a modelling analysis and/or is implemented in a Bayesian feedback system

\section{Response}

We thank Dr Wright for this comment. We believe this issue has been addressed by changing 'computer-guided dosing' to 'model-informed precision dosing' throughout the paper.

\section{Comment 3}

The title seems to promise some debate about whether precision medicine is hype or reality. While the paper presents eight characteristics for precision medicine that are required for "reality", i.e. to inform clinical care, there is only a passing reference to the hype. The title might need some consideration to ensure that it aligns with the content of the paper.

\section{Response}

We thank Dr Wright for this suggestion and we agree. We have now changed the title of the manuscript to represent our position that MIPD is precision medicine technology reality not hype. 


\section{Comment 4}

The eight features are nicely presented but (1) and (3) could be relabelled for clarity. "Defined as precision medicine" is a bit hard to understand on first read. Something like, for example, (1) "aligns to an accepted definition of precision medicine", (3) "Derived from highquality medicine science" are suggestions

\section{Response}

We thank Dr Wright for these suggestions. The titles of both of these sections have been changed accordingly.

Competing Interests: TMP and ARH are both employees of Certara, a global company that provides pharmacometric software and services to the pharmaceutical industry.

The benefits of publishing with F1000Research:

- Your article is published within days, with no editorial bias

- You can publish traditional articles, null/negative results, case reports, data notes and more

- The peer review process is transparent and collaborative

- Your article is indexed in PubMed after passing peer review

- Dedicated customer support at every stage

For pre-submission enquiries, contact research@f1000.com 